\title{
An Efficient Constrained Weighted Least Squares Method with Bias Reduction for TDOA-Based Localization
}

\author{
Liang Zhang, Tao Zhang and Hyo-Sang Shin
}

\begin{abstract}
This paper addresses the source location problem by using time-difference-of-arrival (TDOA) measurements. The twostage weighted least squares (TWLS) algorithm has been widely used in the TDOA location. However, the estimation accuracy of the source location is poor and the bias is significant when the measurement noise is large. Owing to the nonlinear nature of the system model, we reformulate the localization problem as a constrained weighted least squares problem and derive the theoretical bias of the source location estimate from the maximumlikelihood (ML) estimation. To reduce the location bias and improve location accuracy, a novel bias-reduced method is developed based on an iterative constrained weighted least squares algorithm. The new method imposes a set of linear equality constraints instead of the quadratic constraints to suppress the bias. Numerical simulations demonstrate the significant performance improvement of the proposed method over the traditional methods. The bias is reduced significantly and the Cramér-Rao lower bound accuracy can also be achieved.
\end{abstract}

Index Terms - TDOA, Bias reduction, weighted least squares, maximum-likelihood estimation.

\section{INTRODUCTION}

Source location via time-difference-of-arrival (TDOA) measurements has drawn considerable attention thanks to its importance in the applications of sensor networks, radar, and underwater navigation ${ }^{[1][2]}$. Compared with the location method based on the time-of-arrival (TOA), the TDOA-based localization has the advantage that there is no need to synchronize sensor clocks with that of the target ${ }^{[3]}$.

The TDOA-based target localization problem is essentially an optimization problem. It suffers from high nonlinearity and many linear methods have been used in the source location ${ }^{[4]}$. Among the existing methods, the two-stage weighted least squares (TWLS) algorithm is well known for its low computational complexity and proven approximate efficiency ${ }^{[5]}$. In recent years, some improved methods have been proposed.

Manuscript received xxxx, xxxx; revised xxxx, xxxx; accepted xxxx, xxxx. This work was supported in part by National Natural Science Foundation of China 52071080, the Inertial Technology Key Lab Fund under Grant 614250607011709 ,in part by the Fundamental Research Funds for the Central Universities under Grant 2242020k1G009, Key Laboratory Fund for Underwater Information and Control under Grant 614221805051809, and in part by the Jiangsu Key Laboratory Fund for Green Ship Technology under Grant 2019Z01, Remaining funds cultivation project of National Natural Science Foundation of Southeast University under Grant 9S20172204.
An improved algebraic solution for TDOA localization in the presence of sensor position errors was proposed by Liu et al. ${ }^{[6]}$ An efficient estimator for TDOA-based source localization was proposed $^{[7]}$. It could achieve the Cramer-Rao lower bound (CRLB) accuracy with the minimum number of sensors. Besides, the hybrid systems that combine TDOA and other noisy measurements also draw considerable attention. A twostep least-square location estimator was developed for a hybrid TDOA/angle-of-arrival (AOA) location scheme and it gives a much higher location accuracy than TDOA only location ${ }^{[8]}$. For the frequency difference of arrival (FDOA)/TDOA-based localization system, a weighted least-squares minimization method was employed, which did not require initial solution guesses to obtain a location estimate $\mathrm{e}^{[9]}$. Noroozi et $\mathrm{al}^{[10]}$ proposed an improved algebraic solution using TDOA and FDOA and their solution was proved to be less time-consuming than the traditional method. Passive coherent locator (PCL) is usually used in the radar system. A new fusion strategy was performed at the signal processing level based on the TDOA/PCL measurements ${ }^{[11]}$. The theoretical performance of the above hybrid systems gains achievable over the localization technique using only one kind of measurements. All of these methods can lead to a closed-form solution and attain the CRLB performance at low and moderate noise levels.

However, the performance of the closed-form solution will rapidly degrade as the measurement noise increases. The maximum-likelihood (ML) estimation is optimal for the TDOA-based localization problem. The challenge of the ML estimation lies in its nonlinear and nonconvex nature. One resolution to the problem is the linearization based on Taylor expansion $^{[12]}$. Some efficient iteration-based weighted least squares methods were proposed ${ }^{[13][14]}$. These iteration-based methods heavily depend on the quality of the initial estimate. The convergence of the ML estimation is not guaranteed and a local minimum solution could be attained if bad initial estimates are selected. Another resolution to the high nonlinearity issue

(Corresponding author: Tao Zhang.) L. Zhang, T. Zhang are with the School of Instrument Science and Engineering, the Key Laboratory of Micro-Inertial Instrument and Advanced Navigation Technology, Ministry of Education, Southeast University, Nanjing 210096, China (e-mail: zhangliang418@seu.edu.cn; zhangtao22@seu.edu.cn;); H.-S. Shin is with the Institute of Aerospace Sciences, SATM, Cranfield University, MK43 0AL Cranfield,U.K., E-mail: (h.shin@cranfield.ac.uk); 
in the ML problem is the use of the Lagrange multiplier technique ${ }^{[15][16][17]}$. It achieves remarkably better performance than the TWLS approach especially for the higher measurement noise level ${ }^{[15]}$. Convex relaxation methods are also applied to the TDOA-based source location problem ${ }^{[18][19][20]}$. They generally outperform the closed-form solution methods when the noise is considerable. Besides, the semidefinite programming (SDP) methods combined with reformulation linearization $(\mathrm{RLT})^{[21]}$ and the mixed SDP/ second-order cone program $(\mathrm{SOCP})^{[22]}$ were proposed to improve the location accuracy. Both of the methods show superior performance over the TWLS method. However, the SDP method is more costly in terms of computational complexity.

According to the analysis in [23], the noisy measurement will lead to a bias in the closed-form solution. It is necessary to reduce the bias to improve the estimation accuracy when the measurement noise is large. The weighted total least squares (TLS) technique was used to handle the noise correlation between the regressor and regressand ${ }^{[24][25]}$. The bias can be reduced significantly but the estimation variance is higher than the original solution. In 2012, two methods, namely, "BiasSub" and "BiasRed", were proposed ${ }^{[26]}$. Some researchers follow the idea of [26] and bias-reduced methods considering the sensor errors were proposed ${ }^{[27][28]}$. A bias-reduced nonlinear weighted least squares (WLS) method was proposed in [29]. It derived the bias of the WLS solution and subtracted it from the solution.

Generally speaking, the bias-reduced methods can be divided into two types: direct deviation refinement (DDR) and constrained deviation refinement (CDR). DDR method, such as "BiasSub" and the method in [29], requires the covariance matrix of the measurements to be known perfectly and the bias is subtracted from the WLS solution. CDR method, such as "BiasRed", only needs to know the structure of the noise. Thus, "BiasRed" is more practical in the TDOA-based location. To our knowledge, the BiasRed method still suffers from the nonlinear nature of the ML problem. The framework of the BiasRed method is the same as TWLS. It is essentially a twostage weighted least squares method. The difference is that a quadratic constraint is imposed on the first stage in the BiasRed method. The rest of the algorithm is the same as that of the TWLS. The result from the first stage will have bad performance when the measurement noise is large and the biasreduced method will lead to a worse solution. To mitigate the issue, we will explore a method to deal with the problem of the nonlinear nature and the bias simultaneously.

This paper proposes a new bias-reduced method. The bias of the ML has been investigated in many papers ${ }^{[29][30][31]}$. We will first analyze the theoretical bias of the ML problem. Note that the derivation of theoretical bias here is different from that of [30], in which the measurement noise is considered. Then, an iterative constrained weighted least-squares algorithm is developed to handle the nonlinear and bias-reduced problem.

Our goal is to improve the performance of the TDOA-based localization in the case of large measurement noise. The main contributions of the paper are twofold. One is that we derive the theoretical bias of the source location estimate from the ML estimation. The other is that we propose a bias-reduced method, which outperforms the traditional methods especially in the case of large measurement noise.

The structure of the paper is as follows. The first section is the introduction and includes the current research status and the contributions of the paper. The second section analyzes the TDOA positioning method and bias. The third section gives the detailed derivation of the bias-reduced method. The fourth section verifies the effectiveness of the proposed algorithm through simulation. The last section presents summary and future prospects. The main symbols and notations used are shown bellows.

TABLE I

NOMENCLATURE

\begin{tabular}{|c|c|}
\hline Symbol & Explanation \\
\hline M & Number of sensors \\
\hline $\boldsymbol{s}_{i}$ & Position of the ith sensor \\
\hline $\mathbf{u}_{o}$ & The true source location \\
\hline $\mathrm{N}$ & The system dimension \\
\hline$r_{i 1}$ & $\begin{array}{l}\text { Range difference between sensor } i \text { and the } \\
\text { reference sensor }\end{array}$ \\
\hline$r_{i}$ & Range between sensor $\mathrm{i}$ and the source \\
\hline$n$ & TDOA noise vector \\
\hline$n_{i 1}$ & Measurement noise with respect to $r_{i 1}$ \\
\hline$Q$ & Covariance matrix of $\boldsymbol{n}$ \\
\hline$\widehat{u}_{0}$ & The estimated source location \\
\hline CRLB & the Cramer-Rao lower bound \\
\hline $\operatorname{tr}(*)$ & The trace of the matrix * \\
\hline $\mathrm{E}(*)$ & the expectation of parameter * \\
\hline$I_{\mathrm{NxN}}$ & The identity matrix with dimension of $\mathrm{N}$ \\
\hline$\partial *$ & Differential of the parameter $*$ \\
\hline$\odot$ & The element-by-element product \\
\hline$w$ & the weighted matrix \\
\hline$\Delta *$ & The matrix $*$ with noise term \\
\hline$J$ & Cost function of the WLS formulation \\
\hline $\boldsymbol{B}_{1}$ & $\begin{array}{l}\text { Coefficient matrix relating the noise to the } \\
\text { residual vector }\end{array}$ \\
\hline$h, h_{1}$ & Regressand of the WLS formulation \\
\hline$G, G_{1}$ & Regressor of the WLS formulation \\
\hline$y, \theta, V$ & Augmented vector \\
\hline$Z$ & Augmented matrix \\
\hline$\lambda$ & Lagrange multiplier \\
\hline
\end{tabular}

\section{TDOA POSITIONING ANALYSIS}

\section{A. System model}

Consider a scenario of $M$ passive sensors with known positions to locate the source position in $N$-dimensional $(N=2$ or 3 ) space. The position of the $i$ th sensor is known and denoted by $\boldsymbol{s}_{i}(i=1,2,3, \ldots M)$. The source location is unknown and denoted by $\boldsymbol{u}_{o}$. In general, the first sensor $\left(\boldsymbol{s}_{1}\right)$ is usually selected as the reference. The actual TDOA measurement, denoted by $r_{i 1}$, between sensor pairs $i$ and 1 is

$$
r_{i 1}=r_{i}-r_{1}+n_{i 1}
$$

where $r_{i}=\left\|\boldsymbol{u}_{o}-\boldsymbol{s}_{i}\right\|,(i=2,3, \ldots M) . n_{i 1}$ is the measurement 
noise. It is assumed that the TDOA noise vector $\boldsymbol{n}=$ $\left[\begin{array}{lll}n_{21} & \cdots & n_{i 1}\end{array}\right]$ is zero-mean Gaussian distributed with covariance matrix $\boldsymbol{Q}=E_{n}\left(\boldsymbol{n} \boldsymbol{n}^{\boldsymbol{T}}\right)$. Note that $r_{i 1}$ is actually the range difference which is the TDOA multiplied by the known signal propagation speed $\mathrm{c}$. We shall use time differences and range differences interchangeably throughout the paper as they are differed by a constant scaling factor.

According to the analysis in [5], the squared distance between the source and sensor $i$ can be simplified as

$$
\begin{gathered}
r_{i 1}^{2}+\left\|\boldsymbol{s}_{1}\right\|^{2}-\left\|\boldsymbol{s}_{i}\right\|^{2}+2\left(\boldsymbol{s}_{i}-\boldsymbol{s}_{1}\right)^{T} \boldsymbol{u}_{o}+2 r_{i 1}\left\|\boldsymbol{u}_{o}-\boldsymbol{s}_{1}\right\| \\
=2\left\|\boldsymbol{u}_{o}-\boldsymbol{s}_{i}\right\| n_{i 1}+n_{i 1}^{2}
\end{gathered}
$$

Taking all the TDOA measurements into consideration, (2) can be extended as follows.

$$
\begin{gathered}
\boldsymbol{h}-\boldsymbol{G} \boldsymbol{y}=\boldsymbol{B}_{1} \boldsymbol{n}+\boldsymbol{n} \odot \boldsymbol{n} \\
\text { where } \boldsymbol{h}=\left[\begin{array}{c}
r_{21}^{2}+\left\|\boldsymbol{s}_{1}\right\|^{2}-\left\|\boldsymbol{s}_{2}\right\|^{2} \\
\vdots \\
r_{M 1}^{2}+\left\|\boldsymbol{s}_{1}\right\|^{2}-\left\|\boldsymbol{s}_{M}\right\|^{2}
\end{array}\right], \\
\boldsymbol{G}=-2\left[\begin{array}{cc}
\left(\boldsymbol{s}_{2}-\boldsymbol{s}_{1}\right)^{T} & r_{21} \\
\vdots & \vdots \\
\left(\boldsymbol{s}_{M}-\boldsymbol{s}_{1}\right)^{T} & r_{M 1}
\end{array}\right], \boldsymbol{y}=\left[\begin{array}{ll}
\boldsymbol{u}_{o}^{T} & r_{1}
\end{array}\right]^{\boldsymbol{T}}, \\
\boldsymbol{B}_{1}=2 \operatorname{diag}\left(\left[\begin{array}{lll}
r_{2} & \ldots & r_{M}
\end{array}\right]\right) .
\end{gathered}
$$

and " $\odot$ " denotes the element-by-element product.

Let the weighted matrix $\boldsymbol{W}=\left(\boldsymbol{B}_{1} \boldsymbol{Q} \boldsymbol{B}_{1}^{T}\right)^{-1}$ and define the cost function $J(y)$ as follows.

$$
J(\boldsymbol{y}) \triangleq(\boldsymbol{h}-\boldsymbol{G} \boldsymbol{y})^{T} \boldsymbol{W}(\boldsymbol{h}-\boldsymbol{G} \boldsymbol{y})
$$

In (4), the unknown parameter in $\boldsymbol{B}_{1}$ can not be obtained in advance. We adopt an iterative approach. The matrix $\boldsymbol{B}_{\mathbf{1}}$ is set as an identity matrix. Then a rough solution $\boldsymbol{y}$ can be obtained With the solution $\boldsymbol{y}$, then $\boldsymbol{B}_{1}$ and $\boldsymbol{W}$ can be determined. In general, obtaining an approximation of the weighted matrix $\boldsymbol{W}$ is sufficient to obtain the exact final solution. As the minimum cost function (4) is not sensitive to the noise in the weighted matrix.

Considering the relationship between $\boldsymbol{u}_{o}$ and $r_{1}$, (4) can be reformulated as a constrained optimization problem.

$$
\begin{gathered}
\min (\boldsymbol{h}-\boldsymbol{G} \boldsymbol{y})^{T} \boldsymbol{W}(\boldsymbol{h}-\boldsymbol{G} \boldsymbol{y}) \\
\text { s.t. } r_{1}=\left\|\boldsymbol{u}_{o}-\boldsymbol{s}_{1}\right\|
\end{gathered}
$$

\section{B. Constrained weighted least squares solution}

A general solution to (5) is the two-stage weighted least squares $^{[5]}$. However, it suffers from high nonlinearity and large measurement noise. A constrained weighted least-squares solution will be analyzed.

To be able to combine the constraint with the cost function in (5), $\left(\boldsymbol{u}_{o}-\boldsymbol{s}_{1}\right)$ in the constraint should be treated as one vector. The first estimated parameter in $\boldsymbol{y}$ should be changed from $\boldsymbol{u}_{o}$ to $\boldsymbol{u}_{o}-\boldsymbol{s}_{1}$.

Thus, we rewrite (3) as

$$
h_{1}-G_{1} \theta=B_{1} n+n \odot n
$$

where $\boldsymbol{\theta}=\left[\begin{array}{ll}\left(\boldsymbol{u}_{o}-\boldsymbol{s}_{1}\right)^{T} & r_{1}\end{array}\right]^{T}$.

$$
\begin{gathered}
\boldsymbol{h}_{\mathbf{1}}=\left[\begin{array}{c}
r_{21}^{2}-\left(\boldsymbol{s}_{2}-\boldsymbol{s}_{1}\right)^{T}\left(\boldsymbol{s}_{2}-\boldsymbol{s}_{1}\right) \\
\vdots \\
r_{M 1}^{2}-\left(\boldsymbol{s}_{M}-\boldsymbol{s}_{1}\right)^{T}\left(\boldsymbol{s}_{M}-\boldsymbol{s}_{1}\right)
\end{array}\right] \\
\boldsymbol{G}_{\mathbf{1}}=-2\left[\begin{array}{cc}
\left(\boldsymbol{s}_{2}-\boldsymbol{s}_{1}\right)^{T} & r_{21} \\
\vdots & \vdots \\
\left(\boldsymbol{s}_{M}-\boldsymbol{s}_{1}\right)^{T} & r_{M 1}
\end{array}\right]
\end{gathered}
$$

Then, the optimization problem given in (5) can be reformulated as follows.

$$
\begin{gathered}
J(\boldsymbol{\theta})=\min \left(\boldsymbol{h}_{\mathbf{1}}-\boldsymbol{G}_{\mathbf{1}} \boldsymbol{\theta}\right)^{T} \boldsymbol{W}\left(\boldsymbol{h}_{\mathbf{1}}-\boldsymbol{G}_{\mathbf{1}} \boldsymbol{\theta}\right) \\
\text { s.t. } \boldsymbol{\theta}^{\boldsymbol{T}} \boldsymbol{\Sigma} \boldsymbol{\theta}=0
\end{gathered}
$$

Where $\boldsymbol{\Sigma}=\operatorname{diag}(1 \quad 1 \quad-1) .(N=2)$

or $\boldsymbol{\Sigma}=\operatorname{diag}\left(\begin{array}{llll}1 & 1 & 1 & -1\end{array}\right) .(N=3)$

We apply the Lagrange multiplier technique to find the solution to the optimization problem given in (7). It can be reformulated as follows.

$$
L(\boldsymbol{\theta}, \lambda)=\left(\boldsymbol{h}_{\mathbf{1}}-\boldsymbol{G}_{\mathbf{1}} \boldsymbol{\theta}\right)^{T} \boldsymbol{W}\left(\boldsymbol{h}_{\mathbf{1}}-\boldsymbol{G}_{\mathbf{1}} \boldsymbol{\theta}\right)+\lambda \boldsymbol{\theta}^{\boldsymbol{T}} \boldsymbol{\Sigma} \boldsymbol{\theta}
$$

The estimate of $\boldsymbol{\theta}$ is obtained by differentiating $L(\boldsymbol{\theta}, \lambda)$ respect to $\boldsymbol{\theta}$ and then equating the results to zero:

$$
\frac{\partial L(\boldsymbol{\theta}, \lambda)}{\partial \boldsymbol{\theta}}=2\left(\boldsymbol{G}_{1}^{T} \boldsymbol{W} \boldsymbol{G}_{\mathbf{1}}+\lambda \boldsymbol{\Sigma}\right) \boldsymbol{\theta}-2 \boldsymbol{G}_{1}^{T} \boldsymbol{W} \boldsymbol{h}_{\mathbf{1}}=\mathbf{0}
$$

The solution to (9) is

$$
\widehat{\boldsymbol{\theta}}=\left(\boldsymbol{G}_{1}^{T} \boldsymbol{W} \boldsymbol{G}_{\mathbf{1}}+\lambda \boldsymbol{\Sigma}\right)^{-1} \boldsymbol{G}_{1}^{T} \boldsymbol{W} \boldsymbol{h}_{1}
$$

where $\widehat{\boldsymbol{\theta}}$ is the estimated solution of (9).

$\boldsymbol{G}_{1}^{T} \boldsymbol{W} \boldsymbol{G}_{\mathbf{1}}+\lambda \boldsymbol{\Sigma}$ is a symmetric matrix. Substituting (10) into the constraint $\boldsymbol{\theta}^{\boldsymbol{T}} \boldsymbol{\Sigma} \boldsymbol{\theta}=0$ yields

$$
\begin{aligned}
& \boldsymbol{h}_{\mathbf{1}}^{T} \boldsymbol{W}_{\mathbf{1}}^{\boldsymbol{T}} \boldsymbol{G}_{\mathbf{1}}\left(\boldsymbol{G}_{1}^{T} \boldsymbol{W} \boldsymbol{G}_{\mathbf{1}}+\lambda \boldsymbol{\Sigma}\right)^{-1} \boldsymbol{\Sigma}\left(\boldsymbol{G}_{1}^{T} \boldsymbol{W} \boldsymbol{G}_{\mathbf{1}}+\lambda \boldsymbol{\Sigma}\right)^{-1} \boldsymbol{G}_{1}^{T} \boldsymbol{W} \boldsymbol{h}_{\mathbf{1}} \\
&=\boldsymbol{h}_{\mathbf{1}}^{\boldsymbol{T}} \boldsymbol{W}^{T} \boldsymbol{G}_{\mathbf{1}} \boldsymbol{\Sigma}^{-\mathbf{1}}\left(\boldsymbol{G}_{1}^{T} \boldsymbol{W} \boldsymbol{G}_{\mathbf{1}} \boldsymbol{\Sigma}^{-\mathbf{1}}\right. \\
&+\lambda \boldsymbol{I})^{-1}\left(\boldsymbol{G}_{1}^{T} \boldsymbol{W} \boldsymbol{G}_{\mathbf{1}} \boldsymbol{\Sigma}^{-\mathbf{1}}+\lambda \boldsymbol{I}\right)^{-1} \boldsymbol{G}_{1}^{T} \boldsymbol{W} \boldsymbol{h}_{\mathbf{1}} \\
&=0
\end{aligned}
$$

where $\boldsymbol{I}$ is a $(N+1) \times(N+1)$ identity matrix

$\lambda$ is calculated by eigenvalue factorization method and $\boldsymbol{G}_{1}^{T} \boldsymbol{W} \boldsymbol{G}_{\mathbf{1}} \boldsymbol{\Sigma}^{-\mathbf{1}}$ can be factorized as follows.

$$
\boldsymbol{G}_{1}^{T} \boldsymbol{W} \boldsymbol{G}_{\mathbf{1}} \boldsymbol{\Sigma}^{-\mathbf{1}}=\boldsymbol{U} \boldsymbol{\Lambda} \boldsymbol{U}^{-1}
$$

where $\boldsymbol{\Lambda}=\operatorname{diag}\left(\eta_{1} \quad \cdots \quad \eta_{N+1}\right)$.

Substituting (12) into (11), the polynomial equation concerning $\lambda$ can be obtained as follows.

$$
f(\lambda)=\sum_{i=1}^{N+1} \frac{p_{i} q_{i}}{\left(\lambda+\eta_{i}\right)^{2}}
$$

where $\boldsymbol{p}=\left[\begin{array}{lll}p_{1} & \cdots & p_{N+1}\end{array}\right]=\boldsymbol{U}^{T} \boldsymbol{\Sigma}^{-\boldsymbol{T}} \boldsymbol{G}_{1}^{T} \boldsymbol{W} \boldsymbol{h}_{1}$ and $\boldsymbol{q}=\left[\begin{array}{lll}q_{1} & \ldots & q_{N+1}\end{array}\right]=\boldsymbol{U}^{-1} \boldsymbol{G}_{1}^{T} \boldsymbol{W} \boldsymbol{h}_{\mathbf{1}}$.

It can be efficiently solved by finding the roots of a polynomial equation ${ }^{[15]}$. Substituting the real $\lambda$ into (10) provides the estimated value of $\boldsymbol{\theta}$, which is the optimal solution that minimize $J(\boldsymbol{\theta})$ while satisfying the constraint.

\section{Bias analysis}

According to the analysis in [23], the bias is mainly caused by two factors. One is the nonlinearity issue in the ML problem and the other is the noisy measurement. Both of them will be considered in the paper.

The detailed derivation process is shown in the appendix.

The bias of the constrained weighted least-squares solution is as follows.

$$
E_{n}\left(\Delta \boldsymbol{u}_{\mathbf{0}}\right)=E_{n}(\boldsymbol{\alpha})+E_{n}(\boldsymbol{\beta})
$$

where $E_{n}(\boldsymbol{\alpha})$ is given by (44) and $E_{n}(\boldsymbol{\beta})$ is given by (48)

From (14), the bias of the constrained weighted least-squares solution is significant when the measurement noise is large.

To improve the positioning accuracy, the estimation bias should be reduced.

\section{ITERATIVE CONSTRAINED WEIGHTED LEAST SQUARES METHOD FOR BIAS REDUCTION}

The bias of the ML problem is theoretically analyzed in 
section II. In the case of large measurement noise, the bias is significant. We will introduce a novel bias-reduced method, which is different from the existing methods.

The original closed-form solution minimizes

$$
\begin{gathered}
\min \left(\boldsymbol{h}_{\mathbf{1}}-\boldsymbol{G}_{\mathbf{1}} \boldsymbol{\theta}\right)^{T} \boldsymbol{W}\left(\boldsymbol{h}_{\mathbf{1}}-\boldsymbol{G}_{\mathbf{1}} \boldsymbol{\theta}\right) \\
\text { s.t. } \boldsymbol{\theta}^{\boldsymbol{T}} \boldsymbol{\Sigma} \boldsymbol{\theta}=0
\end{gathered}
$$

We introduce an augmented matrix $\boldsymbol{Z}$ and $\boldsymbol{V}$.

$$
\begin{gathered}
\boldsymbol{Z}=\left[\begin{array}{ll}
-\boldsymbol{G}_{1} & \boldsymbol{h}_{1}
\end{array}\right] \\
\boldsymbol{V}=\left[\begin{array}{ll}
\boldsymbol{\theta}^{\boldsymbol{T}} & 1
\end{array}\right]^{T}
\end{gathered}
$$

$\boldsymbol{Z}$ contains measurement noise and it is decomposed as.

$$
\boldsymbol{Z}=\Delta \boldsymbol{Z}+\boldsymbol{Z}^{0}
$$

where $\boldsymbol{Z}^{0}$ is a matrix without any measurement noise. $\Delta \boldsymbol{Z}$ is the noise term, which can be expressed as follows.

$$
\begin{aligned}
& \Delta \boldsymbol{Z}=2\left[\begin{array}{lll}
\mathbf{0}_{(M-1) \times N} & \boldsymbol{n} & \widetilde{\boldsymbol{B}}_{1} \boldsymbol{n}
\end{array}\right] \\
& \widetilde{\boldsymbol{B}}_{1}=\operatorname{diag}\left(r_{21} \quad \ldots \quad r_{i 1}\right)
\end{aligned}
$$

Substituting (18) into (15) yields the cost function

$$
J(\boldsymbol{\theta})=\boldsymbol{V}^{T} \boldsymbol{Z}^{0^{T}} \boldsymbol{W} \boldsymbol{Z}^{0} \boldsymbol{V}+\boldsymbol{V}^{T} \Delta \boldsymbol{Z}^{T} \boldsymbol{W} \Delta \boldsymbol{Z} \boldsymbol{V}+2 \boldsymbol{V}^{T} \Delta \boldsymbol{Z}^{T} \boldsymbol{W} \boldsymbol{Z}^{0} \boldsymbol{V} \text { (19) }
$$

The third term $2 \boldsymbol{V}^{T} \Delta \boldsymbol{Z}^{T} \boldsymbol{W} \boldsymbol{Z}^{0} \boldsymbol{V}$ vanishes in the expectation because $\Delta \boldsymbol{Z}$ is zero-mean. Take the expectation of $J(\boldsymbol{\theta})$ to obtain the cost function on the average.

$$
E[J(\boldsymbol{\theta})]=\boldsymbol{V}^{T} \boldsymbol{Z}^{0^{T}} \boldsymbol{W} \boldsymbol{Z}^{0} \boldsymbol{V}+\boldsymbol{V}^{T} E\left[\Delta \boldsymbol{Z}^{T} \boldsymbol{W} \Delta \boldsymbol{Z}\right] \boldsymbol{V}
$$

We regard the second term on the right-hand as a constant constraint to the cost function. Thus, we find $\boldsymbol{V}$ by

$$
\begin{gathered}
\min \boldsymbol{V}^{T} \boldsymbol{Z}^{T} \boldsymbol{W} \boldsymbol{Z} \boldsymbol{V} \\
\text { s.t. } \boldsymbol{V}^{T} \boldsymbol{\Omega} \boldsymbol{V}=k
\end{gathered}
$$

where $\boldsymbol{\Omega}=E\left[\Delta \boldsymbol{Z}^{T} \boldsymbol{W} \Delta \boldsymbol{Z}\right]$ and constant $\mathrm{k}$ can be any value.

$$
\begin{array}{r}
\boldsymbol{\Omega}=E\left[\Delta \boldsymbol{Z}^{T} \boldsymbol{W} \Delta \boldsymbol{Z}\right] \\
=\left[\begin{array}{cc}
\mathbf{0}_{N \times N} & \mathbf{0}_{N \times 2} \\
\mathbf{0}_{2 \times N} & \widetilde{\mathbf{\Omega}}
\end{array}\right]
\end{array}
$$

and

$$
\widetilde{\mathbf{\Omega}}=4\left[\begin{array}{cc}
\operatorname{tr}(\boldsymbol{W} \boldsymbol{Q}) & \operatorname{tr}\left(\boldsymbol{W} \widetilde{\boldsymbol{B}}_{\mathbf{1}} \boldsymbol{Q}\right) \\
\operatorname{tr}\left(\widetilde{\boldsymbol{B}}_{\mathbf{1}} \boldsymbol{W} \boldsymbol{Q}\right) & \operatorname{tr}\left(\widetilde{\boldsymbol{B}}_{\mathbf{1}} \boldsymbol{W} \widetilde{\boldsymbol{B}}_{\mathbf{1}} \boldsymbol{Q}\right)
\end{array}\right]
$$

The traditional bias-reduced method is based on (21). The solution to (21) neglects the constraint relationship between $\boldsymbol{u}_{o}$ and $r_{1}$ in the first stage. A second step to reduce the nonlinear error is necessary. The drawback of the traditional method is that the source location in the first stage suffers from the measurement noise and an inaccurate result will lead to a local minimum solution in the second stage.

Thus, an iterative approximation method is developed to solve the bias-reduction problem. The new iterative technique follows the idea given in [32]. But a different solution to the iterative approximation problem is designed in the paper, which takes the constant constraint caused by the noisy measurements into consideration.

According to (7) and (21), it can be obtained

$$
\begin{gathered}
\min \boldsymbol{V}^{T} \boldsymbol{Z}^{T} \boldsymbol{W} \boldsymbol{Z} \boldsymbol{V} \\
\text { s.t. } \boldsymbol{V}^{T} \boldsymbol{\Omega} \boldsymbol{V}=k \\
\boldsymbol{V}^{\boldsymbol{T}} \boldsymbol{\Sigma}_{\mathbf{1}} \boldsymbol{V}=0
\end{gathered}
$$

where $\left\{\begin{array}{c}\boldsymbol{\Sigma}_{\mathbf{1}}=\operatorname{diag}\left(\left[\begin{array}{lllll}1 & 1 & -1 & 0\end{array}\right) \quad N=2\right. \\ \boldsymbol{\Sigma}_{\mathbf{1}}=\operatorname{diag}\left(\left[\begin{array}{lllll}1 & 1 & 1 & -1 & 0\end{array}\right]\right) \quad N=3\end{array}\right.$

By using Lagrange multiplier $\lambda$, we obtain the auxiliary cost function

$$
\begin{gathered}
\min \boldsymbol{V}^{T} \boldsymbol{Z}^{T} \boldsymbol{W Z V}+\lambda\left(k-\boldsymbol{V}^{T} \boldsymbol{\Omega} \boldsymbol{V}\right) \\
\text { s.t. } \boldsymbol{V}^{T} \boldsymbol{\Sigma}_{\mathbf{1}} \boldsymbol{V}=0
\end{gathered}
$$

It is difficult to obtain a globally optimal solution. If we replace one of the variable $\boldsymbol{V}$ with a known estimate $\widehat{\boldsymbol{V}}$, the nonconvex constraint becomes a linear equality constraint.

$$
\begin{gathered}
\min \boldsymbol{V}^{T} \boldsymbol{Z}^{T} \boldsymbol{W} \boldsymbol{Z} \boldsymbol{V}+\lambda\left(k-\boldsymbol{V}^{T} \boldsymbol{\Omega V}\right) \\
\text { s.t. } \widehat{\boldsymbol{V}}^{\boldsymbol{T}} \boldsymbol{\Sigma}_{\mathbf{1}} \boldsymbol{V}=0
\end{gathered}
$$

(26) shows the main difference between the proposed method and the traditional method. The linear equality constraint in (26) can be reformulated as follows.

$$
P V=0
$$

where $\boldsymbol{P}=\widehat{\boldsymbol{V}}^{\boldsymbol{T}} \boldsymbol{\Sigma}_{\mathbf{1}}$ and $\widehat{\boldsymbol{V}}$ is a known vector.

Based on the generalized inverse theory of a matrix [33], the solution to (27) can be obtained as follows

$$
\boldsymbol{V}=\left(\boldsymbol{I}-\boldsymbol{P}^{+} \boldsymbol{P}\right) \boldsymbol{\xi}
$$

where $\xi \in \boldsymbol{R}^{N+1}$ is any vector. $\boldsymbol{P}^{+}=\boldsymbol{P}^{T}\left(\boldsymbol{P} \boldsymbol{P}^{T}\right)^{-1}$.

The general solution to (27) can be expressed as

$$
\boldsymbol{V}=\boldsymbol{U} \xi
$$

where $\boldsymbol{U}=\left(\boldsymbol{I}-\boldsymbol{P}^{+} \boldsymbol{P}\right)$.

Substituting (29) into (25) yields the cost function

$$
J(\xi)=\min (\boldsymbol{U} \xi)^{T} \boldsymbol{Z}^{T} \boldsymbol{W} \boldsymbol{Z} \boldsymbol{U} \boldsymbol{\xi}+\lambda\left(k-(\boldsymbol{U} \xi)^{T} \boldsymbol{\Omega} \mathbf{U} \xi\right)
$$

It is an ML problem and the optimal solution $\xi$ satisfies

$$
\frac{\partial J(\xi)}{\partial \xi}=2 \boldsymbol{U}^{T} \boldsymbol{Z}^{T} \boldsymbol{W Z U} \xi-2 \lambda \boldsymbol{U}^{T} \boldsymbol{\Omega} \mathbf{U} \xi=0
$$

We have the following relationship

$$
\boldsymbol{U}^{T} \boldsymbol{Z}^{T} \boldsymbol{W Z U} \boldsymbol{\xi}=\lambda \boldsymbol{U}^{T} \boldsymbol{\Omega U} \boldsymbol{\xi}
$$

We need to minimize $\lambda$. Through generalized singular value decomposition (GSVD) theory, the optimal solution $\xi$ is the eigenvector that corresponds to the minimum generalized eigenvalue of the pair $\left(\begin{array}{ll}\boldsymbol{U}^{T} \boldsymbol{Z}^{T} \boldsymbol{W} \boldsymbol{Z} \boldsymbol{U} & \left.\boldsymbol{U}^{T} \boldsymbol{\Omega} \mathbf{U}\right) .\end{array}\right.$

When $\xi$ is obtained, substitute it into (29) and we will have the optimal solution $\boldsymbol{V}$.

From the above discussion, we finish the derivation of the bias-reduced method. However, there are two problems with the above method.

a) Since $r_{i}$ in the matrix $\boldsymbol{B}_{\mathbf{1}}$ is unknown, $\boldsymbol{B}_{\mathbf{1}}$ cannot be obtained.

b) The initial value $\widehat{V}$ in (26) is important and it can not be obtained.

To solve the above problems, we adopt an iterative approach. The matrix $\boldsymbol{B}_{\mathbf{1}}$ is set as an identity matrix. Then a rough solution $\boldsymbol{\theta}$ can be obtained through the method in Section II. In general, obtaining an approximation of the weighted matrix $\mathbf{W}$ is sufficient to obtain the exact final solution. As the minimum cost function (7) is not sensitive to the noise in the weighted matrix.

When the rough value of $\boldsymbol{\theta}$ is obtained, the matrix $\boldsymbol{B}_{\mathbf{1}}$ can be obtained as follows.

$$
\left\{\begin{array}{c}
\boldsymbol{B}_{1}=2 \operatorname{diag}\left(\left[\begin{array}{lll}
r_{2} & \ldots & r_{i}
\end{array}\right]\right) \\
r_{i}=\left\|\boldsymbol{\theta}(1: N)+\boldsymbol{s}_{1}-\boldsymbol{s}_{i}\right\| \quad i=2, \ldots M
\end{array}\right.
$$

The rough value can be selected as an initial value in (26). The iterative constrained weighted least squares method is formally presented in Algorithm 1.

Sometimes, the iterative method cannot guarantee convergence to the global solution of the ML problem. A divergence factor $\sigma$ is adopted in the iterative method. If the equation diverges, the estimated value will be far from the initial estimate and we will take the solution in section II-B as step 8 shows. 
Algorithm 1: A bias-reduced method based on iterative constrained weighted least squares

Step 1. Set $\boldsymbol{B}_{\mathbf{1}}=\boldsymbol{I}_{\boldsymbol{M - 1}}$ and solve the constraint WLS optimization problem (7) by (9)-(13). A rough value $\boldsymbol{\theta}^{\mathbf{0}}$ can be obtained.

Step 2. Initialize $k=0$. Define a convergence threshold $\varepsilon$, a divergence threshold $\sigma$ and a maximum number of iterations $\tau$.Reformulate the weighting matrix $\mathbf{W}$ with $\boldsymbol{\theta}^{\mathbf{0}}$. Set the initial value $\widetilde{\boldsymbol{\theta}}^{\boldsymbol{k}}=\boldsymbol{\theta}^{\mathbf{0}}$ and $\widehat{\boldsymbol{V}}=$ $\left[\begin{array}{ll}\widetilde{\boldsymbol{\theta}}^{\boldsymbol{k}} & 1\end{array}\right]^{T}$

Step 3. Set $k=k+1$. formulate the approximate linear constraint WLS optimization problem (26) with $\widehat{\boldsymbol{V}}$.

Step 4. Solve the approximate problem (30) based on GSVD to obtain the estimated value of $\xi$.

Step 5. Obtain the estimate $\widetilde{\boldsymbol{\theta}}^{\boldsymbol{k}+\mathbf{1}}$ with $\boldsymbol{\xi}$ according to (29).

Step 6. Reformulate the weighting matrix $\mathbf{W}$ with $\widetilde{\boldsymbol{\theta}}^{\boldsymbol{k}+\mathbf{1}}$. Set $\widehat{\boldsymbol{V}}=$ $\left[\begin{array}{ll}\widetilde{\theta}^{k+1} & 1\end{array}\right]^{T}$.

Step 7. If $\left\|\widetilde{\boldsymbol{\theta}}^{\boldsymbol{k}+\mathbf{1}}-\widetilde{\boldsymbol{\theta}}^{\boldsymbol{k}}\right\|<\varepsilon$ or $\mathrm{k}>\tau$, go to step 9, otherwise, go to step 3 .

Step 8 If $\left\|\widetilde{\boldsymbol{\theta}}^{\boldsymbol{k}+\mathbf{1}}-\boldsymbol{\theta}^{\mathbf{0}}\right\|>\sigma$, set $\widetilde{\boldsymbol{\theta}}^{\boldsymbol{k}+\mathbf{1}}=\boldsymbol{\theta}^{\mathbf{0}}$.

Step 9. $\boldsymbol{u}_{o}=\widetilde{\boldsymbol{\theta}}^{\boldsymbol{k}+\mathbf{1}}(1: N)+\boldsymbol{s}_{1}$.

\section{SIMULATION TEST AND ANALYSIS}

To verify the effectiveness of the proposed algorithm, simulations are performed in this section. We apply the proposed algorithm to two localization scenarios and the results are compared with that of several existing methods. 1000 Monte Carlo simulations are performed for each test.

Symbols used for the simulations are as follows:

1) 'TWLS' denotes the two-step weighted least squares algorithm described in [5].

2) 'BiasRed' denotes the bias-reduced method described in [26].

3) 'L-WLS' denotes the Lagrange- weighted least squares described in section II without reducing the bias.

4) 'Proposed method' denotes the proposed bias-reduced method described in section $\mathrm{V}$.

5) 'TheoryBias' denotes the theoretical bias norm calculated by $\left\|\Delta u_{\text {theory }}\right\|$ and $\Delta u_{\text {theory }}$ is obtained from (14).

Note: The CDR method has an advantage over the DDR method in that it only requires the structure of $\mathbf{Q}^{[26]}$. The 'BiasRed' and 'The proposed' both belong to the CDR method. Thus, the method in [26] and the proposed method are mainly used for comparison.

The localization accuracy is evaluated in terms of the root mean square error (RMSE) and the bias norm of the source position, which is defined as follows.

$$
\begin{gathered}
\text { RMSE }=\sqrt{\frac{\sum_{i=1}^{L}\left\|\widehat{\boldsymbol{u}}_{o}-\boldsymbol{u}_{o}\right\|^{2}}{L}} \\
\text { BiasNorm }=\left\|\frac{\sum_{i=1}^{L} \widehat{\boldsymbol{u}}_{o}}{L}-\boldsymbol{u}_{o}\right\|
\end{gathered}
$$

where $\boldsymbol{u}_{o}$ denotes the true source position. $\mathrm{L}=1000$ is the number of ensemble runs.

The TDOA noise is Gaussian and its covariance matrix is equal to the noise power times $\boldsymbol{Q}=\left(\boldsymbol{I}_{N}+\mathbf{1}_{N} \mathbf{1}_{N}^{T}\right) / 2$. At a given
SNR, the noise power is obtained as follows with the signal propagation speed $\mathrm{sec}=3 \times 10^{8} \mathrm{~m} / \mathrm{s}$.

$$
\sigma_{\delta}^{2}=\frac{1}{8 \pi^{2} \operatorname{SNR}\left(16 \times 10^{18}\right)} \sec ^{2}
$$

\section{A. Scenario 1-The impact of the measurement noise}

We will compare the location performance under the condition of different measurement noise. We consider the sensor-source geometry, where the sensor network has an array of eight sensors and their positions are given by $s_{i}=$ $\left[12 \cos \left(\frac{\pi}{6}(i-2)\right) \quad 12 \sin \left(\frac{\pi}{6}(i-2)\right)\right]^{T},(i=2, \ldots 8)$. The reference sensor is located at $s_{1}=\left[\begin{array}{ll}0 & 0\end{array}\right]^{T}$. The source is located at $u_{0}=\left[250 \cos \left(\frac{\pi}{16}\right) \quad 250 \sin \left(\frac{\pi}{16}\right)\right]^{T}$. The noise power is varied from -15 to $10 \mathrm{~dB}$.

Comparison of the RMSE and bias norm with different measurement noise is shown in Fig. 1 and Fig. 2.

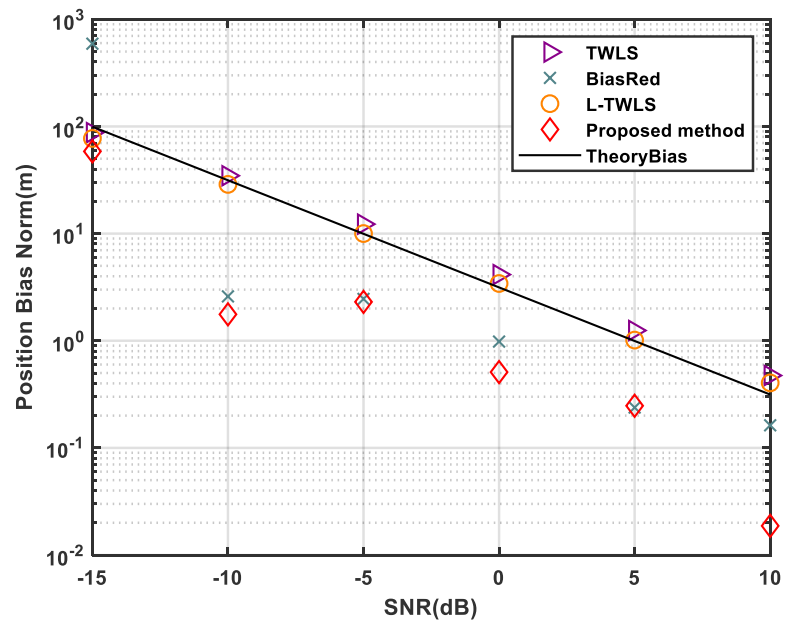

Fig. 1 Comparison of bias norm in the TDOA source location

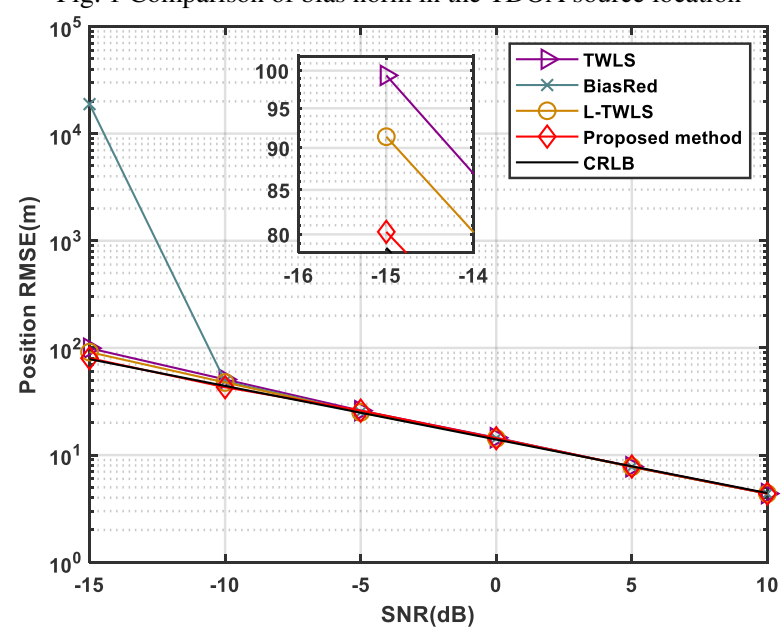

Fig. 2 Comparison of the RMSE with different measurement noise.

In Fig. 1, the black line is the theoretical bias calculated by (28). Fig. 1 indicates the bias of the location increases with the decrease of SNR. The bias of the BiasRed and the proposed method can be significantly reduced. For example, when SNR $=-10 \mathrm{~dB}$, the bias norm from BiasRed and the proposed method is $2.60 \mathrm{~m}$ and $1.76 \mathrm{~m}$ respectively. The proposed method outperforms the BiasRed method in terms of bias-reduction in the given condition. When $\mathrm{SNR}=-15 \mathrm{~dB}$, the bias norm from 
BiasRed is larger than the theoretical bias norm. However, the bias norm of the proposed method is still the lowest among these methods. In Fig. 2, the black line is the trace of the CRLB. It indicates the proposed method achieves the CRLB accuracy very well no matter what value is the SNR. The proposed method has the smallest RMSE among all the methods, e.g., when $\mathrm{SNR}=-15 \mathrm{~dB}$, the RMSE of the proposed method is $80.3 \mathrm{~m}$, which is smaller than the TWLS (99.4m), BiasRed (18847.7m), and L-TWLS (91.4m). It has an 11.1-m reduction in RMSE as compared with the L-TWLS. The RMSE of BiasRed rapidly increases when $\mathrm{SNR}=-15 \mathrm{~dB}$. It indicates the BiasRed method suffers from the large measurement noise. The result is consistent with the analysis in section $\mathrm{V}$. Thus, the proposed method has better performance.

Fig. 3 compares the theoretical and simulation bias norm values. The actual bias norm is from the L-TWLS method. The bias theoretically found matches very well with the actual bias norm. It can be seen in the figure that the proposed method can reduce the bias significantly, e.g., when the SNR is $-10 \mathrm{~dB}$, the proposed method has a $27-\mathrm{m}$ reduction in bias compared to the L-TWLS method. It demonstrates the superb performance of the proposed bias reduction procedure.

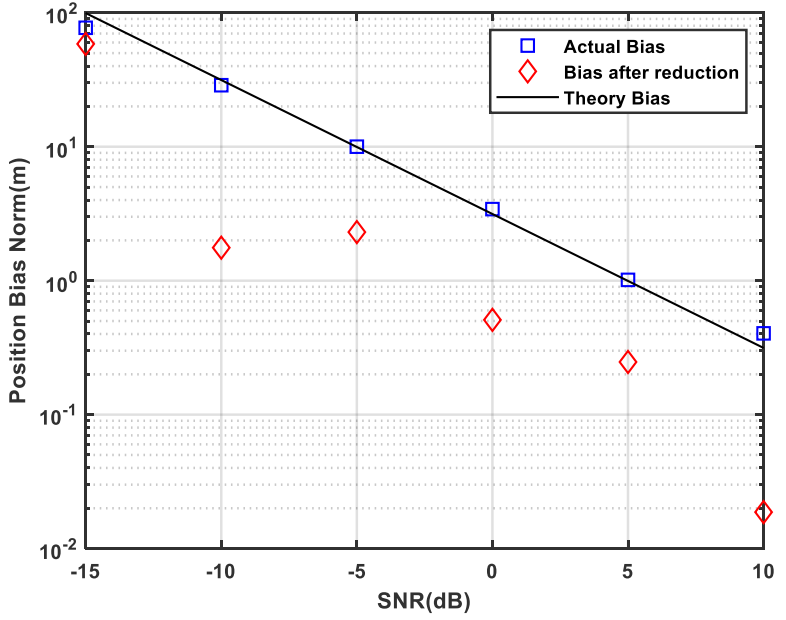

Fig. 3 Theoretical and simulation bias values of TDOA source location estimate from the proposed method

The proposed method is superior to the traditional methods especially in the condition of large measurement noise. It can reduce the bias significantly and achieve the CRLB accuracy very well.

\section{B. Scenario 2-The impact of the sensor numbers}

This section compares the location performance under the condition of different sensor numbers. The sensor network has an array of several sensors and their positions are given by $s_{i}=$ $\left[12 \cos \left(\frac{\pi}{6}(i-2)\right) 12 \sin \left(\frac{\pi}{6}(i-2)\right)\right]^{T}$. In this scenario, the sensor number $(i)$ is varied from 8 to 12 . The reference sensor is located at $s_{1}=\left[\begin{array}{ll}0 & 0\end{array}\right]^{T}$. The source is located at $u_{0}=$ $\left[350 \cos \left(\frac{\pi}{16}\right) \quad 350 \sin \left(\frac{\pi}{16}\right)\right]^{T}$. The noise power is set as $-6 \mathrm{~dB}$.

A comparison of the RMSE and bias norm with different sensor numbers are shown in Fig. 4 and Fig. 5.

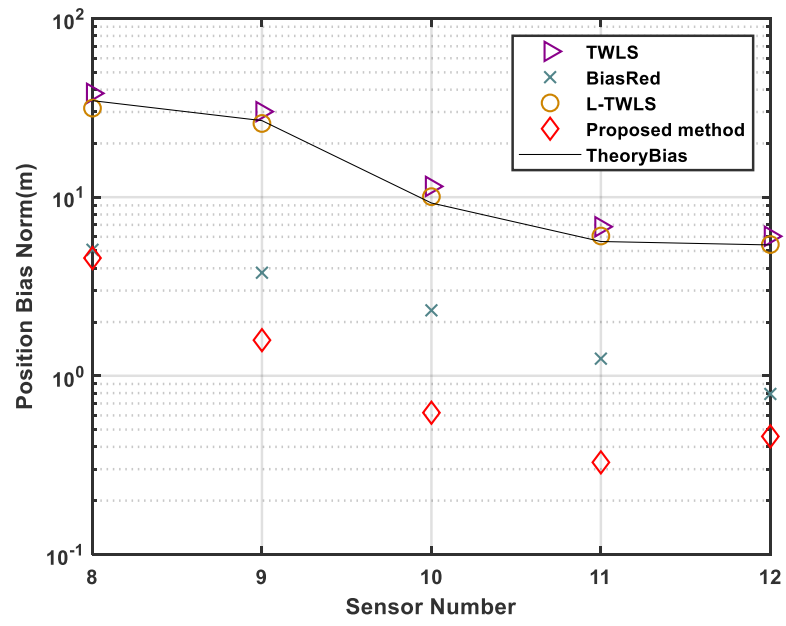

Fig. 4 Comparison of bias norm in TDOA source location estimates from different solution methods

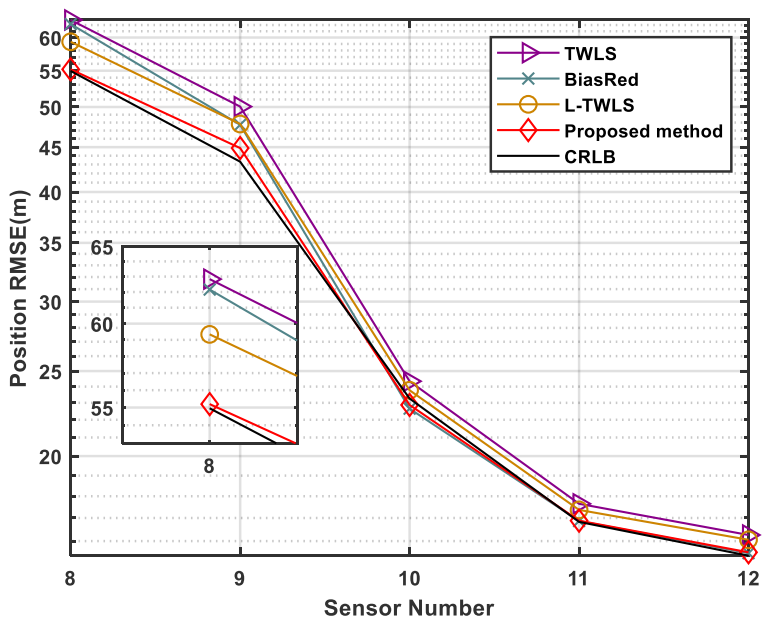

Fig. 5 Comparison of the RMSE with different sensor numbers

Fig. 4 compares the bias norm of the proposed method and other methods. The bias of the BiasRed and the proposed method can be significantly reduced. However, the bias from the BiasRed method is larger than that of the proposed method. For example, when the sensor number is 9 , the bias norm from BiasRed and the proposed method is $3.78 \mathrm{~m}$ and $1.58 \mathrm{~m}$ respectively. Fig. 5 compares the RMSE of the proposed method and other methods. All the algorithms perform exhibit reasonable performance when the sensor number is large. The proposed method achieves the CRLB performance best no matter how the sensor number changes. However, traditional methods such as TWLS, BiasRed, and L-TWLS cannot achieve the CRLB performance when the sensor number decreases. In comparison, the proposed method provides a more stable performance both in terms of RMSE and bias.

Fig. 6 compares the theoretical and simulation bias norm values. The actual bias norm is from the L-TWLS method. The theoretical bias norm matches very well with the actual bias norm. It can be seen in the figure that the proposed method can reduce the bias significantly, e.g., when the sensor number is 9 , the proposed method has a $24.29-\mathrm{m}$ reduction in bias. 


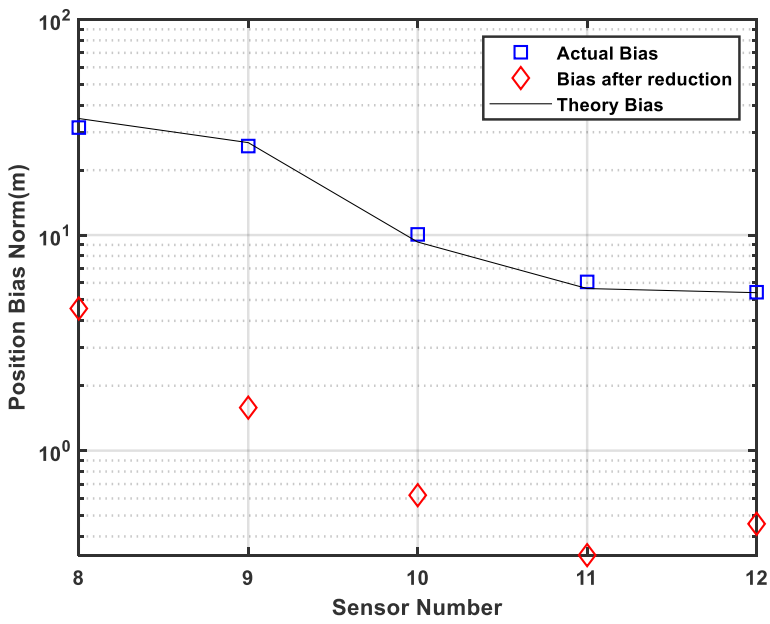

Fig. 6 Theoretical and simulation bias values of TDOA source location estimate from the proposed method

From the above figures, the proposed method has a more stable performance both in bias-reduction and RMSE. The RMSE of the proposed method is always closer to the CRLB accuracy.

To analyze the overall computational complexity of these methods, the running times of different methods are compared. In general, the higher the computational complexity, the longer the running time.

The average running times of different methods as sensor number varies are compared. The average running times, using MATLAB in a personal computer with core (TM) i5-4460, and the main frequency is $3.2 \mathrm{GHz}$ are plotted in Fig. 7 .

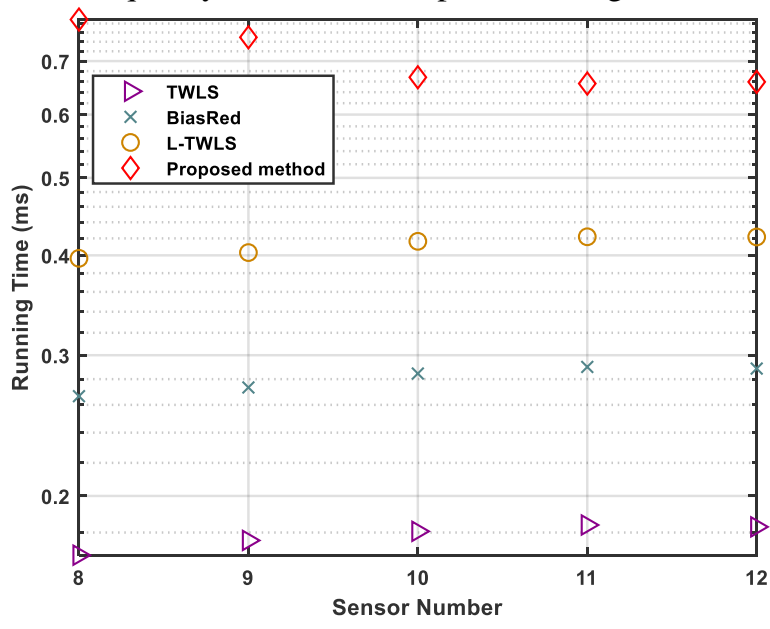

Fig. 7 Comparison of the average running times of different methods

It can be seen from Fig. 7 that the traditional TWLS method requires the least running time but its estimation accuracy is the worst. The proposed method and the traditional BiasRed method require the same order of magnitude of running time, that is, around $0.7 \mathrm{~ms}$ and $0.3 \mathrm{~ms}$, respectively. Although the proposed method requires the most running time, its estimation accuracy is the highest. In the engineering aspect, if the algorithms are implemented on some chips with high configurations, which will meet the requirements of the computational efficiency, the proposed method is a suitable choice.

\section{CONCLUSION}

We have presented a novel bias-reduced method in the paper. The bias of the ML problem is theoretically analyzed in the paper. Traditional bias-reduced methods suffer from the high nonlinearity of the ML problem and the noisy measurement. The CDR methods are usually based on the assumption that the estimation parameters are irrelevant and a further step is carried out to reduce the nonlinear error. However, the error will rapidly increase if the measurement noise is large. The proposed method can handle the issue with the problem of the nonlinear nature and the bias simultaneously and it is more robust as it tolerates better noisy measurements. Several scenarios have been illustrated to verify the effectiveness of the proposed algorithm. Our algorithm outperforms traditional methods especially in the condition of large measurement noise. It can reduce the bias significantly and achieve the CRLB accuracy very well.

Although the study proves to be more effective than traditional methods when the measurement noise changes, there are still many challenges in its practical application. Our future work includes the robust localization method in the presence of TDOA measurement outliers and the successful application on the real scenarios, such as the underwater passive navigation ${ }^{[2]}$.

\section{APPENDIX}

According to (7), the ML problem can be expressed as follows.

$$
J(\boldsymbol{\theta})=\min \left(\boldsymbol{h}_{\mathbf{1}}-\boldsymbol{G}_{\mathbf{1}} \boldsymbol{\theta}\right)^{T} \boldsymbol{W}\left(\boldsymbol{h}_{\mathbf{1}}-\boldsymbol{G}_{\mathbf{1}} \boldsymbol{\theta}\right)
$$

The solution $\widehat{\boldsymbol{u}}_{\mathbf{0}}$ satisfies

$$
P\left(\widehat{\boldsymbol{u}}_{\mathbf{0}}\right)=\left.\frac{\partial J(\boldsymbol{\theta})}{\partial \boldsymbol{u}_{\mathbf{0}}}\right|_{\boldsymbol{u}_{\mathbf{0}}=\widehat{\boldsymbol{u}}_{\mathbf{0}}}=0
$$

Due to the nonlinear nature of the estimation problem, the Taylor series expansion of $P\left(\widehat{\boldsymbol{u}}_{\mathbf{0}}\right)$ at $\boldsymbol{u}_{\mathbf{0}}$ is

$$
P\left(\widehat{\boldsymbol{u}}_{\mathbf{0}}\right) \triangleq \frac{\partial J(\boldsymbol{\theta})}{\partial \boldsymbol{u}_{\mathbf{0}}}+\frac{\partial^{2} J(\boldsymbol{\theta})}{\partial \boldsymbol{u}_{\mathbf{0}} \partial \boldsymbol{u}_{\mathbf{0}} \boldsymbol{T}}\left(\widehat{\boldsymbol{u}}_{\mathbf{0}}-\boldsymbol{u}_{\mathbf{0}}\right)=0
$$

Form (35), $\frac{\partial J(\boldsymbol{\theta})}{\partial \boldsymbol{u}_{\mathbf{0}}}$ can be obtained as

$$
\frac{\partial J(\boldsymbol{\theta})}{\partial \boldsymbol{u}_{\mathbf{0}}}=-2 \boldsymbol{F}_{1}^{T} \boldsymbol{W}\left(\boldsymbol{h}_{\mathbf{1}}-\boldsymbol{G}_{\mathbf{1}} \boldsymbol{\theta}\right)
$$

where $\boldsymbol{F}_{1}=-2\left[\begin{array}{c}r_{21} \boldsymbol{\rho}^{T}+\left(\boldsymbol{s}_{2}-\boldsymbol{s}_{1}\right)^{T} \\ \vdots \\ r_{M 1} \boldsymbol{\rho}^{T}+\left(\boldsymbol{s}_{M}-\boldsymbol{s}_{1}\right)^{T}\end{array}\right], \boldsymbol{\rho}=\frac{\left(\boldsymbol{u}_{o}-\boldsymbol{s}_{1}\right)}{\left\|\boldsymbol{u}_{o}-\boldsymbol{s}_{1}\right\|}$.

According to (38), $\frac{\partial^{2} J(\boldsymbol{\theta})}{\partial \boldsymbol{u}_{0} \partial \boldsymbol{u}_{0} \boldsymbol{T}}$ can be obtained as follows.

$$
\boldsymbol{H}=\frac{\partial^{2} J(\theta)}{\partial \boldsymbol{u}_{0} \partial \boldsymbol{u}_{0}^{T}}=\boldsymbol{A}-\boldsymbol{B}
$$

where $\boldsymbol{A}=2 \boldsymbol{F}_{1}^{T} \boldsymbol{W} \boldsymbol{F}_{1}$

$$
\begin{aligned}
& \boldsymbol{B}=2\left(\sum_{i=1}^{M-1} \sum_{j=1}^{M-1} \boldsymbol{X}_{i j} n_{j 1} \boldsymbol{f}_{i}+\sum_{i=1}^{M-1} \sum_{j=1}^{M-1} \boldsymbol{W}_{i j} \boldsymbol{\varepsilon}_{j} \boldsymbol{f}_{i}\right) \\
& \boldsymbol{X}=\boldsymbol{W} \boldsymbol{B}_{1}, \boldsymbol{f}_{i}=-2 r_{i 1} \frac{\boldsymbol{I}_{N \times N}-\boldsymbol{\rho} \boldsymbol{\rho}}{\left\|\boldsymbol{u}_{o}-\boldsymbol{s}_{1}\right\|}
\end{aligned}
$$

$\boldsymbol{\varepsilon}$ is a column vector formed by the diagonal elements of $\mathbf{Q}$.

$\boldsymbol{H}^{-1}$ can be approximated as follows ${ }^{[30]}$.

$$
\boldsymbol{H}^{-1}=(\boldsymbol{A}-\boldsymbol{B})^{-1} \approx \boldsymbol{A}^{-1}+\boldsymbol{A}^{-1} \boldsymbol{B} \boldsymbol{A}^{-1}
$$

According to (6), the bias can be expressed as follows. 


$$
\begin{aligned}
\Delta \boldsymbol{u}_{0}=\widehat{\boldsymbol{u}}_{0}-\boldsymbol{u}_{0} \approx & -\boldsymbol{H}^{-1} \frac{\partial \boldsymbol{J}(\theta)}{\partial \boldsymbol{u}_{0}} \\
& =2 A^{-1} \boldsymbol{F}_{1}^{T} W\left(B_{1} n+n \odot n\right) \\
& +2 A^{-1} B A^{-1} F_{1}^{T} W\left(B_{1} n+n \odot n\right) \\
& =\alpha+\beta
\end{aligned}
$$

where $\boldsymbol{\alpha}=2 \boldsymbol{A}^{-1} \boldsymbol{F}_{1}^{T} \boldsymbol{W}\left(\boldsymbol{B}_{1} \boldsymbol{n}+\boldsymbol{n} \odot \boldsymbol{n}\right)$,

$\boldsymbol{\beta}=2 \boldsymbol{A}^{-1} \boldsymbol{B} \boldsymbol{A}^{-1} \boldsymbol{F}_{1}^{T} \boldsymbol{W}\left(\boldsymbol{B}_{1} \boldsymbol{n}+\boldsymbol{n} \odot \boldsymbol{n}\right)$.

Note that $\boldsymbol{F}_{1}$ is correlated to the measurement noise $\boldsymbol{n}$, which should be considered in evaluating the expectation. Thus, we can express

$$
\left\{\begin{aligned}
\boldsymbol{F}_{1} & =\Delta \boldsymbol{F}_{1}^{0}+\boldsymbol{F}_{1}^{0} \\
\boldsymbol{A} & =\Delta \boldsymbol{A}+\boldsymbol{A}^{0}
\end{aligned}\right.
$$

where $\Delta \boldsymbol{A}=\Delta \boldsymbol{F}_{1}^{0^{T}} \boldsymbol{W} \boldsymbol{F}_{1}^{0}+\boldsymbol{F}_{1}^{0^{T}} \boldsymbol{W} \Delta \boldsymbol{F}_{1}^{0}$,

$\boldsymbol{F}_{1}^{0}=-2\left[\begin{array}{c}r_{21}^{0} \boldsymbol{\rho}^{T}+\left(\boldsymbol{s}_{2}-\boldsymbol{s}_{1}\right)^{T} \\ \vdots \\ r_{i 1}^{0} \boldsymbol{\rho}^{T}+\left(\boldsymbol{s}_{i}-\boldsymbol{s}_{1}\right)^{T}\end{array}\right], r_{i 1}^{0}(i=2, \ldots M)$ is the true

TDOA measurement.

$\Delta \boldsymbol{F}_{1}^{0}=-2\left[\begin{array}{c}n_{21} \boldsymbol{\rho}^{T} \\ \vdots \\ n_{2 i 1} \boldsymbol{\rho}^{T}\end{array}\right]=-2 \boldsymbol{n} \boldsymbol{\rho}^{T}$.

First, consider the measurement noise and $\boldsymbol{\alpha}$ can be expressed as follows.

$$
\begin{aligned}
& \alpha=2 D B_{1} n+2 D n \odot n-2 A^{0^{-1}} \Delta A D B_{1} n+ \\
& 2 \boldsymbol{A}^{0^{-1}} \Delta \boldsymbol{F}_{1}^{0^{T}} \boldsymbol{W} \boldsymbol{B}_{1} n
\end{aligned}
$$

where $\mathbf{D}=\boldsymbol{A}^{0^{-1}} \boldsymbol{F}_{1}^{0^{T}} \boldsymbol{W}$.

Thus, the expectation of $\alpha$ is

$$
\begin{aligned}
E_{n}(\boldsymbol{\alpha})=2 \boldsymbol{D} E_{n}[\boldsymbol{n} & \odot \boldsymbol{n}]-2 \boldsymbol{A}^{0^{-1}} E_{n}\left[\Delta \boldsymbol{A D} \boldsymbol{B}_{\mathbf{1}} \boldsymbol{n}\right] \\
& +2 \boldsymbol{A}^{0^{-1}} E_{n}\left[\Delta \boldsymbol{F}_{1}^{0^{T}} \boldsymbol{W} \boldsymbol{B}_{\mathbf{1}} \boldsymbol{n}\right] \\
& =2 \boldsymbol{D} E_{n}[\boldsymbol{n} \odot \boldsymbol{n}] \\
& -2 \boldsymbol{A}^{0^{-1}} \boldsymbol{F}_{1}^{0^{T}} \boldsymbol{W} E_{n}\left[\Delta \boldsymbol{F}_{1}^{0} \boldsymbol{R} \boldsymbol{n}\right] \\
& +2 \boldsymbol{A}^{0^{-1}} E_{n}\left[\Delta \boldsymbol{F}_{1}^{0^{T}} \boldsymbol{S n}\right]
\end{aligned}
$$

where $R=D B_{1}, S=W B_{1}-W F_{1}^{0} D B_{1}$.

Then $E_{n}(\boldsymbol{\alpha})$ can be expressed as follows.

$$
\begin{aligned}
& E_{n}(\boldsymbol{\alpha})=2 \boldsymbol{D} \boldsymbol{\varepsilon}-2 \boldsymbol{A}^{0^{-1}} \boldsymbol{F}_{1}^{0^{T}} \boldsymbol{W} \boldsymbol{\alpha}_{1}+2 \boldsymbol{A}^{0^{-1}} \boldsymbol{\alpha}_{2} \\
& \text { where } \boldsymbol{\alpha}_{1}=-2\left[\begin{array}{c}
\sum_{i=1}^{M-1} \boldsymbol{\rho}^{T} \boldsymbol{R}_{i} \boldsymbol{Q}_{1, i} \\
\vdots \\
\sum_{i=1}^{M-1} \boldsymbol{\rho}^{T} \boldsymbol{R}_{i} \boldsymbol{Q}_{N, i}
\end{array}\right],
\end{aligned}
$$

$\boldsymbol{\alpha}_{2}=-2 \boldsymbol{\rho} \operatorname{Tr}(\boldsymbol{S} \boldsymbol{Q})$

$\boldsymbol{\varepsilon}$ is a column vector formed by the diagonal elements of $\mathbf{Q} \cdot \boldsymbol{R}_{\boldsymbol{i}}$, $\boldsymbol{q}_{i}$ and $\boldsymbol{S}_{\boldsymbol{i}}$ are the $i$ th column of $\boldsymbol{R}, \boldsymbol{S}$, and $\mathbf{Q}$.

Considering the measurement noise, $\boldsymbol{\beta}$ can be expressed as follows.

$$
\begin{gathered}
\boldsymbol{\beta}=2\left(\Delta \boldsymbol{A}+\boldsymbol{A}^{0}\right)^{-1}\left(\boldsymbol{B}^{0}+\Delta \boldsymbol{B}\right)\left(\Delta \boldsymbol{A}+\boldsymbol{A}^{0}\right)^{-1}\left(\Delta \boldsymbol{F}_{1}^{0}+\right. \\
\left.\boldsymbol{F}_{1}^{0}\right)^{T} \boldsymbol{W}\left(\boldsymbol{B}_{\mathbf{1}} \boldsymbol{n}+\boldsymbol{n} \odot \boldsymbol{n}\right)
\end{gathered}
$$

where $\boldsymbol{B}=\boldsymbol{B}^{0}+\Delta \boldsymbol{B}, \Delta \boldsymbol{B}$ is the noise term.

Ignoring the third-order and high-order noise term, $\beta$ can be expressed as.

$$
\boldsymbol{\beta}=2 \boldsymbol{A}^{0^{-1}} \boldsymbol{B}_{x}^{\mathbf{0}} \boldsymbol{A}^{0^{-1}} \boldsymbol{F}_{1}^{0^{T}} \boldsymbol{W} \boldsymbol{B}_{1} \boldsymbol{n}
$$

where $\boldsymbol{B}_{x}^{0}=2 \sum_{i=1}^{M-1} \sum_{j=1}^{M-1} \boldsymbol{X}_{i j} n_{j 1} \boldsymbol{f}_{i}$.

Thus, the expectation of $\beta$ can be expressed as follows.

$$
E_{n}(\boldsymbol{\beta})=4 \boldsymbol{A}^{0^{-1}} \sum_{i=1}^{M-1} \boldsymbol{C}_{i}
$$

where $\boldsymbol{C}_{i}=\sum_{j=1}^{M-1} \boldsymbol{X}_{i j} \boldsymbol{f}_{i} \boldsymbol{A}^{0^{-1}} \boldsymbol{F}_{1}^{0^{T}} \boldsymbol{W} \boldsymbol{B}_{\mathbf{1}} \boldsymbol{Q}(j)$,

$\boldsymbol{Q}(j)$ is the $i$ th column of $\mathbf{Q}$. As the high-order noise term in (46) is neglected, (48) is not related to the noise in the matrix $\boldsymbol{h}_{\mathbf{1}}$ and matrix $\boldsymbol{G}_{\mathbf{1}}$.

From the above analysis, the bias can be expressed as follows.

$$
E_{n}\left(\Delta \boldsymbol{u}_{\mathbf{0}}\right)=E_{n}(\boldsymbol{\alpha})+E_{n}(\boldsymbol{\beta})
$$

\section{REFERENCES}

[1] A. Beck, P. Stoica, and J. Li, "Exact and approximate solutions of source localization problems," IEEE Trans. Signal Process., vol. 56, no. 5, pp. 1770-1778, May 2008.

[2] B. Jin, X. Xu, Y. Zhu, T. Zhang and Q. Fei, "Single-Source Aided SemiAutonomous Passive Location for Correcting the Position of an Underwater Vehicle," in IEEE Sensors Journal, vol. 19, no. 9, pp. 32673275, 1 May, 2019.

[3] M. R. Gholami, S. Gezici and E. G. Strom, "TDOA Based Positioning in the Presence of Unknown Clock Skew," in IEEE Transactions on Communications, vol. 61, no. 6, pp. 2522-2534, June 2013.

[4] A. Noroozi, M. A. Sebt, and A. H. Oveis, "Efficient Weighted Least Squares Estimator for Moving Target Localization in Distributed MIMO Radar With Location Uncertainties," in IEEE Systems Journal, vol. 13, no. 4, pp. 4454-4463, Dec. 2019.

[5] Y. T. Chan and K. C. Ho, "A simple and efficient estimator for hyperbolic location," in IEEE Transactions on Signal Processing, vol. 42, no. 8, pp. 1905-1915, Aug. 1994.

[6] Y. Liu, F. Guo, L. Yang, and W. Jiang, "An Improved Algebraic Solution for TDOA Localization With Sensor Position Errors," in IEEE Communications Letters, vol. 19, no. 12, pp. 2218-2221, Dec. 2015.

[7] R. Amiri, F. Behnia and A. Noroozi, "An Efficient Estimator for TDOABased Source Localization With Minimum Number of Sensors," in IEEE Communications Letters, vol. 22, no. 12, pp. 2499-2502, Dec. 2018.

[8] Li Cong and Weihua Zhuang, "Hybrid TDOA/AOA mobile user location for wideband CDMA cellular systems," in IEEE Transactions on Wireless Communications, vol. 1, no. 3, pp. 439-447, July 2002, doi: 10.1109/TWC.2002.800542.

[9] K. C. Ho and Wenwei Xu, "An accurate algebraic solution for moving source location using TDOA and FDOA measurements," in IEEE Transactions on Signal Processing, vol. 52, no. 9, pp. 2453-2463, Sept. 2004, doi: 10.1109/TSP.2004.831921.

[10] A. Noroozi, A. H. Oveis, S. M. Hosseini and M. A. Sebt, "Improved Algebraic Solution for Source Localization From TDOA and FDOA Measurements," in IEEE Wireless Communications Letters, vol. 7, no. 3, pp. 352-355, June 2018

[11] A. Aubry, V. Carotenuto, A. De Maio and L. Pallotta, "Joint Exploitation of TDOA and PCL Techniques for Two-Dimensional Target Localization," in IEEE Transactions on Aerospace and Electronic Systems, vol. 56, no. 1, pp. 597-609, Feb. 2020, doi: 10.1109/TAES.2019.2917990.

[12] D. J. Torrieri, "Statistical Theory of Passive Location Systems," in IEEE Transactions on Aerospace and Electronic Systems, vol. AES-20, no. 2, pp. 183-198, March 1984.

[13] H. Yu, G. Huang, J. Gao, and B. Liu, "An Efficient Constrained Weighted Least Squares Algorithm for Moving Source Location Using TDOA and FDOA Measurements," in IEEE Transactions on Wireless Communications, vol. 11, no. 1, pp. 44-47, January 2012.

[14] B. Jin; X. Xu; T. Zhang, "Robust Time-Difference-of-Arrival (TDOA) Localization Using Weighted Least Squares with Cone Tangent Plane Constraint. Sensors, vol.18, pp.778,2018.

[15] H. Yu, G. Huang, J. Gao, et al. "Practical constrained least-square algorithm for moving source location using TDOA and FDOA measurements". Journal of Systems Engineering and Electronics, vol.23, no.4, pp.488-494, 2012

[16] V. Heidari, M. Amidzade, K. Sadeghi and A. M. Pezeshk, "Exact solutions of time difference of arrival source localization based on semidefinite programming and Lagrange multiplier: complexity and performance analysis," in IET Signal Processing, vol. 8, no. 8, pp. 868877,102014 
[17] F. Quo and K. C. Ho, "A quadratic constraint solution method for TDOA and FDOA localization," 2011 IEEE International Conference on Acoustics, Speech, and Signal Processing (ICASSP), Prague, 2011, pp. 2588-2591.

[18] G. Wang, Y. Li and N. Ansari, "A Semidefinite Relaxation Method for Source Localization Using TDOA and FDOA Measurements," in IEEE Transactions on Vehicular Technology, vol. 62, no. 2, pp. 853-862, Feb. 2013.

[19] Y.Zou, H. Liu, W. Xie and Q. Wan, "Semidefinite Programming Methods for Alleviating Sensor Position Error in TDOA Localization," in IEEE Access, vol. 5, pp. 23111-23120, 2017.

[20] Y. Zou and H. Liu, "Semidefinite Programming Methods for Alleviating Clock Synchronization Bias and Sensor Position Errors in TDOA Localization," in IEEE Signal Processing Letters, vol. 27, pp. 241-245, 2020.

[21] Z. Zheng, H. Zhang, W. Wang, S. So, Source localization using TDOA and FDOA measurements based on semidefinite programming and reformulation linearization, Journal of the Franklin Institute, vol.356, no.18, pp. 11817-11838, 2019,

[22] G. Wang and K. C. Ho, "Convex Relaxation Methods for Unified NearField and Far-Field TDOA-Based Localization," in IEEE Transactions on Wireless Communications, vol. 18, no. 4, pp. 2346-2360, April 2019.

[23] Y. Ji, C. Yu and B. D. O. Anderson, "Systematic Bias Correction in Source Localization," in IEEE Transactions on Aerospace and Electronic Systems, vol. 49, no. 3, pp. 1692-1709, July 2013.

[24] K. Yang, J. An, Z. Xu and X. Bu, "A Generalized Total Least-Square Algorithm for Hyperbolic Location," 2008 4th International Conference on Wireless Communications, Networking and Mobile Computing, Dalian, pp. 1-4, 2008.

[25] R. J. Barton and D. Rao, "Performance capabilities of long-range UWBIR TDOA localization systems," EURASIP J. Adv. Signal Process., vol. 2008, no.1, 2008

[26] K. C. Ho, "Bias Reduction for an Explicit Solution of Source Localization Using TDOA," in IEEE Transactions on Signal Processing, vol. 60, no. 5, pp. 2101-2114, May 2012.

[27] X. Chen, D. Wang, J. Yin. et al. Bias reduction for TDOA localization in the presence of receiver position errors and synchronization clock bias. EURASIP J. Adv. Signal Process. vol.2019, no.7,2019.

[28] Y. Zhao, Z. Li, B. Hao, J. Si, and P. Wan, "Bias reduced method for TDOA and AOA localization in the presence of sensor errors," 2017 IEEE International Conference on Communications (ICC), Paris, 2017, pp. 1-6.

[29] G. Wang, S. Cai, Y. Li and N. Ansari, "A Bias-Reduced Nonlinear WLS Method for TDOA/FDOA-Based Source Localization," in IEEE Transactions on Vehicular Technology, vol. 65, no. 10, pp. 8603-8615, Oct. 2016.

[30] L. Rui and K. C. Ho, "Bias analysis of maximum likelihood target location estimator," in IEEE Transactions on Aerospace and Electronic Systems, vol. 50, no. 4, pp. 2679-2693, October 2014

[31] L. Rui and K. C. Ho, "Bias analysis of source localization using the maximum likelihood estimator," 2012 IEEE International Conference on Acoustics, Speech and Signal Processing (ICASSP), Kyoto, 2012, pp. 2605-2608

[32] X. Qu, L. Xie, and W. Tan, "Iterative Constrained Weighted Least Squares Source Localization Using TDOA and FDOA Measurements," in IEEE Transactions on Signal Processing, vol. 65, no. 15, pp. 3990-4003, 1 Aug.1, 2017.

[33] A. Ben-Israel and T. N. E. Greville, Generalized Inverses: Theory and Applications, 2nd ed. New York, NY, USA: Wiley, 2002.

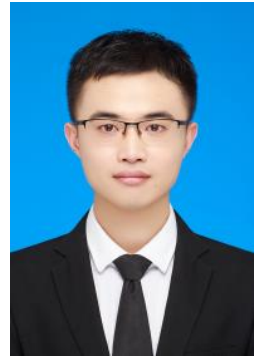

Liang Zhang received the M.S. degree in Navigation, Guidance, and Control from Nanjing University of Aeronautics and Astronautics, Nanjing, Jiangsu, in 2017. He is currently pursuing a Ph.D. degree in instrument science and technology at Southeast University, Nanjing, Jiangsu. His research interest includes inertial navigation, integrated navigation technology, and AUV underwater positioning technology.

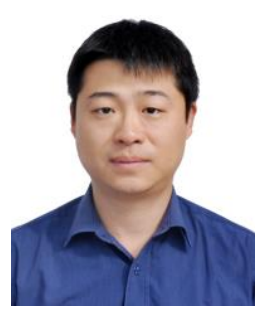

Tao Zhang received the Ph.D. degree in precision instrument and machinery from Southeast University, Nanjing, China, in 2008. He is currently a Professor with the School of Instrument Science and Engineering, Southeast University. His research interests include inertial navigation, AUV underwater positioning, and integrated navigation.

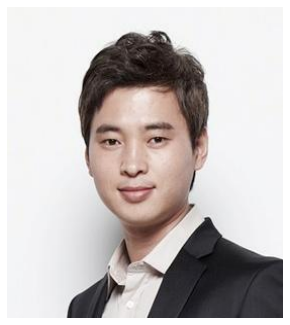

Hyo-Sang Shin received his BSc from Pusan National University in 2004 and gained an MSc on flight dynamics, guidance, and control in Aerospace Engineering from KAIST and a Ph.D. on cooperative missile guidance from Cranfield University in 2006 and 2010, respectively. He is currently a Professor on Guidance, Control and Navigation Systems in Autonomous and Intelligent Systems Group at Cranfield University. His current research interests include multiple target tracking, adaptive and sensor-based control, and distributed control of multiple agent systems. 


\section{An efficient constrained weighted least squares method with bias reduction for TDOA-based localization}

\section{Zhang, Liang}

IEEE

Zhang L, Zhang T, Shin H-S. (2021) An efficient constrained weighted least squares method with bias reduction for TDOA-based localization. IEEE Sensors Journal, Volume 21, Issue 8, April 2021, pp. 10122-10131

https://doi.org/10.1109/JSEN.2021.3057448

Downloaded from Cranfield Library Services E-Repository 